\title{
EFEITO DO ESTÁGIO DE DESENVOLVIMENTO DAS FLORES E DA APLICAÇÃO DE FUNGICIDAS NO CONTROLE DA PODRIDÃO FLORAL DOS CITROS ${ }^{1}$
}

\author{
SÉRGIO RUFFO ROBERTO² \& ANDREY VETORELLI BORGES ${ }^{3}$
}

\begin{abstract}
RESUMO - O presente trabalho teve por objetivo determinar os melhores estágios do desenvolvimento das flores de laranja-doce para o controle da Podridão Floral dos Citros (PFC), avaliando-se diversas épocas de aplicação e doses de fungicidas. Os estudos foram realizados em pomares de laranjas-'Natal' e 'Pêra' (Citrus sinensis [L.] Osbeck), onde foram marcados aleatoriamente ramos florais pertencentes à florada temporã (janeiro de 1994), caracterizando as fases de desenvolvimento de cada botão floral. Em um primeiro ensaio, avaliou-se o controle da doença através de pulverizações manuais, onde foram aplicados os seguintes tratamentos (doses por $1 \mathrm{~L}$ ): a) testemunha; b) benomyl 0,5 g, no dia de marcação dos ramos (aplicação normal); c) benomyl 0,5 g, 3 a 4 dias depois da marcação (aplicação tardia); e d) benomyl 0,5 g em 2 aplicações, uma na marcação dos botões e outra uma semana depois. As aplicações foram feitas com pulverizadores manuais, molhando-se apenas os ramos com flores marcados. Em um segundo ensaio, avaliou-se o controle da doença através de pulverizações tratorizadas. Na laranja-'Natal', estudaram-se os seguintes tratamentos (doses por $2.000 \mathrm{~L}$ ): a) testemunha; b) benomyl $1 \mathrm{~kg} \mathrm{em} \mathrm{4/jan;} \mathrm{c)} \mathrm{benomyl} 1 \mathrm{~kg} \mathrm{em} 4$ e 11/jan; e d) benomyl $1 \mathrm{~kg}$ em 8/jan. Na laranja'Pêra', os seguintes tratamentos foram aplicados (doses por $2.000 \mathrm{~L}$ ): a) testemunha; b) benomyl $1 \mathrm{~kg}, 4 / \mathrm{jan}$; c) benomyl $1 \mathrm{~kg}, 4 \mathrm{e} 10 /$ jan; d) benomyl $1 \mathrm{~kg}+$ captan 3,5 kg, 4/jan; e) benomyl 0,75 kg + captan 2,5 kg, 4/jan; f) benomyl $1 \mathrm{~kg}+$ captan 2,5 kg, 4/jan; g) benomyl $1 \mathrm{~kg}+$ captan 2,5 kg, 4/jan + benomyl $1 \mathrm{~kg}, 10 / \mathrm{jan}$; h) benomyl 1,5 kg, 4/jan; e i) benomyl 1,5 kg, 4 e 10/jan. Avaliaram-se, em intervalos de dois dias, a freqüência e a época de ocorrência das lesões nas pétalas dos botões florais, a porcentagem de pegamento e o índice de cálices retidos de cada parcela. Pelos resultados, pôde-se concluir que o benomyl é eficiente no controle da PFC em doses de 0,5-0,75 g. $\mathrm{L}^{-1}$, e sua resposta podem ser obtida em floradas temporãs tratadas nos estágios de predominância de botões redondos brancos até o período anterior à antese.
\end{abstract}

Termos para indexação: citrus, pegamento de frutos, Colletotrichum acutatum.

\section{FLOWER BUD STAGE AND FUNGICIDE APPLICATION EFFECT CONTROL ON POSTBLOOM FRUIT DROP OF CITRUS}

\begin{abstract}
This research was an attempt to determine the most appropriate flower bud stages of sweet orange in which fungicide application is more effective to control postbloom fruit drop disease. The trials were carried out in a 'Natal' and 'Pera' sweet orange orchards (Citrus sinensis [L.] Osbeck). Flower branches of summer bloom (January, 1994) were randomly marked and the different stages of development of buds were characterized. In a first trial, the disease control was evaluated through manual spraying. The following treatments were applied (doses per $1 \mathrm{~L}$ ): a) control; b) benomyl $0.5 \mathrm{~g}$, when buds were marked (regular application); c) benomyl $0.5 \mathrm{~g}, 3-4$ days after the treatment $\mathrm{b}$ (late application), and d) benomyl $0.5 \mathrm{~g}$ in 2 applications (regular application and 7 days later). In a second trial, the disease control was evaluated through regular spraying, using a tractor and speed sprayer. On 'Natal' orchard, the following treatments were applied (doses per 2,000 L): a) control; b) benomyl $1 \mathrm{~kg}$ on Jan $4^{\text {th }}$; c) benomyl $1 \mathrm{~kg}$ on Jan $4^{\text {th }}$ and $11^{\text {th}}$; and d) benomyl $1 \mathrm{~kg}$ on Jan $8^{\text {th }}$. On 'Pera' the following treatments were applied (doses per 2,000 L): a) control; b) benomyl $1 \mathrm{~kg}$, Jan, $4^{\text {th }} ; \mathrm{c}$ ) benomyl $1 \mathrm{~kg}$, Jan, $4^{\text {th }}$ and $10^{\text {th }}$; d) benomyl $1 \mathrm{~kg}+$ captan $3.5 \mathrm{~kg}$, Jan, $4^{\text {th }}$ ); e) benomyl $0.75 \mathrm{~kg}+$ captan $2.5 \mathrm{~kg}, \mathrm{Jan}$, $4^{\text {th }}$; f) benomyl $1 \mathrm{~kg}+$ captan $2.5 \mathrm{~kg}$, Jan, $4^{\text {th }} ;$ g) benomyl $1 \mathrm{~kg}+$ captan $2.5 \mathrm{~kg}$, Jan, $4^{\text {th }}+$ benomyl $1 \mathrm{~kg}$, Jan, $\left.10^{\text {th }} ; \mathrm{h}\right)$ benomyl $1.5 \mathrm{~kg}$, Jan, $4^{\text {th }}$; and i) benomyl $1.5 \mathrm{~kg}$, Jan, $4^{\text {th }}$ and $10^{\text {th }}$. Disease lesions on petals, fruit set and incidence of persistent calyx parameters were evaluated every other day. Through the results, it was possible to conclude that benomyl is efficient to control the disease in doses ranging from 0.5-0.75 g. $\mathrm{L}^{-1}$, and its response may be obtained in summer blooms when round to elongate bud stages are prevalent.
\end{abstract}

Index terms: citrus, fruit set, Colletotrichum acutatum.

1 Trabalho $n^{0}$ 273/2000. Recebido: 21/12/2000. Aceito para publicação: 27/06/2001.

2 Eng. Agr. Dr. Professor Adjunto. Área de de Fruticultura. Universidade Estadual de Londrina - UEL. Centro de Ciências Agrárias. Departamento de Agronomia. Caixa Postal 6001.86051-990. Londrina - PR. e-mail: sroberto@uel.br

3 Acadêmico do Curso de Graduação em Agronomia. Universidade Estadual de Londrina - UEL. Centro de Ciências Agrárias. Departamento de Agronomia. Caixa Postal 6001. 86051-990. Londrina - PR. 


\section{INTRODUÇÃO}

A Podridão Floral dos Citros (PFC) é uma doença das flores de citros causada por Colletotrichum acutatum, também conhecida por "Queda Prematura de Frutos Jovens" (Fagan, 1979; Goes \& Creste, 2000). A doença é conhecida no Brasil pelo menos desde 1977 em laranjas, e há muito mais tempo em limas ácidas. Os sintomas iniciais mais evidentes são lesões alaranjadas nas pétalas de flores abertas (Timmer et al., 1994). A queda das pétalas é então retardada e muitas secam ainda retidas no cálice. Freqüentemente, observa-se a necrose do pistilo, iniciando-se no estigma e descendo pelo estilo. Crescimentos fúngicos alaranjados podem também ser vistos nos estiletes dos estames, no estigma, estilo, ou no nectário. A abscisão do estilo dos frutinhos também é retardada (Feichtenberger et al., 1997).

A aparência dos frutos recém-formados ao final da florada é geralmente normal. Eles, no entanto, tornam-se rapidamente pálidos ou amarelados, não caem, mas também não se desenvolvem. Lentamente, no entanto, a maioria dos frutinhos destaca-se, separando-se pela zona de abscisão à base do ovário, e deixando persistente na planta a "estrelinha", ou cálice retido (Agostini \& Timmer, 1994).

A doença ocorre em função de chuvas durante a florada (Feichtenberger et al., 1997). Com os invernos pouco rigorosos, e com as chuvas precoces dos últimos anos, a PFC tem sido responsável por perdas significativas desde 1989 nas regiões ao sul de Araraquara-SP, e desde $1992 \mathrm{em}$ todo o Estado. Isto se deve ao fato de o inóculo ter permanecido alto de ano para ano e as floradas de primavera terem ocorrido mais cedo que o normal em alguns anos, juntamente com as chuvas.

A PFC foi registrada em quase toda a citricultura das Américas na década de 80 (Denham, 1979). Métodos de controle biológico (Moretto, 2000) e principalmente químicos, utilizandose dediversos fungicidas, foram testados para seu controle, e os melhores resultados vieram das aplicações de benzimidazóis (Timmer \& Zitko, 1992; Sonoda, 1993). Coutinho et al. (1994) determinaram que os melhores resultados de controle da doença são obtidos quando as flores se encontram nos estágios de botão redondo branco até completamente desenvolvido. Entretanto, uma grande dificuldade são as diferentes sucessões de flores, tendo em vista que os benzimidazóis não se comportam como sistêmicos nas pétalas, pois estas não possuem estômatos funcionais. No Estado de São Paulo, no entanto, o sucesso do controle tem sido variável. Problemas como épocas de controle, doses do produto, número e intervalo de aplicações, e tipos de pulverização precisam ser mais bem estudados, o que motivou a pesquisa.

Os objetivos deste trabalho foram: a) acompanhar o desenvolvimento de botões florais de laranja-doce pulverizados ou não, sob condições favoráveis à doença, com a finalidade de determinar os melhores estágios para o controle da doença; b) determinar os estágios de desenvolvimento floral mais suscetíveis à infecção; e c) avaliar o controle através de pulverizações de diversos fungicidas em diferentes concentrações e misturas em florada temporã.

\section{MATERIAL E MÉTODOS}

Pulverizações manuais. Plantas de laranja-'Natal'
(Citrus sinensis [L.] Osbeck) sobre limão- 'Cravo' (Citrus limonia Osbeck) (plantio de 1986, espaçamento 9 × 6 m, Rincão, SP), e de laranja-'Pêra' (C. sinensis [L.] Osbeck) sobre limão- 'Cravo' (C. limonia Osbeck) (plantio de 1978, espaçamento $8 \times 5,5 \mathrm{~m}$, Gavião Peixoto-SP), foram utilizadas nos experimentos. Foram marcados aleatoriamente ramos florais pertencentes à florada temporã, nos dias 3 ('Natal') e 4 ('Pêra') de janeiro de 1994. Ambos os pomares tiveram ataques intensos de PFC na florada da primavera (setembro/outubro de 1993), possuindo uma safra extremamente reduzida e um grande número de cálices retidos.

O delineamento experimental utilizado foi o de blocos casualizados, com 25 repetições. Cada ramo de flores marcado representou uma parcela. Foram testados 4 tratamentos (dose por $1 \mathrm{~L}$ ): a) testemunha; b) benomyl $0,5 \mathrm{~g}$, no dia de marcação dos ramos (aplicação normal); c) benomyl 0,5 g, 3 a 4 dias depois da marcação (aplicação tardia); e d) benomyl $0,5 \mathrm{~g}$ em 2 aplicações, uma na marcação dos botões e outra uma semana depois. As aplicações foram feitas com pulverizadores manuais, molhando-se apenas os ramos com flores marcadas até o ponto de escorrimento.

Nestes experimentos, os botões florais foram mapeados em cada ramo marcado, para seu acompanhamento. Foram convencionados os seguintes estágios florais (abreviaturas em parênteses) (Figura 1): botão fechado verde (V); botão fechado redondo branco $(\mathbf{R}) ;(\mathbf{1 / 4})$ e (1/2) de cotonete - tamanhos do botão floral em relação ao seu desenvolvimento final, conhecido como "cotonete"; cotonete $(\mathbf{C})$; aberto $(\mathbf{A B})$; chumbinho $(\mathbf{C H})$; e estilo caído (EC).

Pulverizações com atomizadores. Nos mesmos talhões dos experimentos anteriores, e na mesma florada temporã, foram pulverizados fungicidas em 1 ou 2 aplicações, usando equipamentos tratorizados. Na laranja-'Natal' foram utilizados pulverizadores Rolanzir, com saída de calda unilateral, na velocidade de $1,5 \mathrm{~km} / \mathrm{h}$, com volume de $6 \mathrm{~L} /$ planta. O delineamento experimental foi o de blocos casualizados, com 3 repetições e 4 tratamentos (doses por $2.000 \mathrm{~L}$ ): a) testemunha; b) benomyl $1 \mathrm{~kg}$, em 4/jan; c) benomyl $1 \mathrm{~kg}$, em 4 e 11/jan; e d) benomyl $1 \mathrm{~kg}$, em 8/jan. Foram marcados 20 ramos com flores aleatoriamente em cada parcela para acompanhamento do desenvolvimento dos botões florais.

Na laranja-'Pêra', foram utilizados pulverizadores Rolanzir, com saída de calda unilateral, na velocidade de $5 \mathrm{~km} /$ $\mathrm{h}$, com volume de $8 \mathrm{~L} /$ planta. O delineamento experimental foi o de blocos casualizados, com 3 repetições e 9 tratamentos: (doses por $2.000 \mathrm{~L}$ ): a) testemunha; b) benomyl $1 \mathrm{~kg}$, 4/jan; c) benomyl $1 \mathrm{~kg}, 4$ e 10/jan; d) benomyl $1 \mathrm{~kg}+$ captan $3,5 \mathrm{~kg}, 4 / \mathrm{jan}$; e) benomyl $0,75 \mathrm{~kg}+$ captan $2,5 \mathrm{~kg}, 4 / \mathrm{jan}$; f) benomyl $1 \mathrm{~kg}+$ captan $2,5 \mathrm{~kg}, 4 / \mathrm{jan} ; \mathrm{g}$ ) benomyl $1 \mathrm{~kg}+$ captan $2,5 \mathrm{~kg}$, 4/jan + benomyl $1 \mathrm{~kg}, 10 / \mathrm{jan}$; h) benomyl 1,5 kg, 4/jan; e i) benomyl 1,5 kg, $4 \mathrm{e}$ $10 /$ jan. Foram pulverizadas faixas de plantas no talhão com florada uniforme, e 20 ramos com flores marcadas em cada faixa.

Em todos os experimentos descritos anteriormente (pulverizações manuais e com atomizadores), avaliaram-se a cada intervalo de dois dias, a freqüência e época de ocorrência das lesões nas pétalas dos botões florais e do estilo necrosado, a porcentagem de pegamento e o índice de cálices retidos de cada parcela, bem como a evolução dos estágios dos botões durante o seu desenvolvimento. As avaliações iniciaram-se na primeira aplicação dos fungicidas e estenderam-se até a observação do pegamento efetivo dos frutos. A partir destes dados, foi analisado 
o efeito das aplicações dos fungicidas sobre a PFC através da análise de variância, e a separação das médias foi feita pelo teste de Duncan, a 5\% de probabilidade, no programa SAS (Statistical Analysis System) versão 6.14.

\section{RESULTADOS E DISCUSSÃO}

Pulverizações manuais. O clima foi extremamente favorável à incidência de $\mathrm{PFC}$, com chuvas quase diárias durante toda a florada temporã. A distribuição dos estágios dos botões florais no início dos tratamentos mostrou a predominância de botões redondos brancos fechados na laranja- 'Natal', e de botões totalmente desenvolvidos, mas ainda fechados (cotonetes) na 'Pêra'.

Todos os tratamentos com benomyl resultaram em redução do número de flores com sintomas de estilos necrosados, de cálices retidos e com significativo aumento no pegamento de frutos (Tabelas 1 e 2). As aplicações em plantas molhadas, seguidas de chuvas leves, resultaram em melhor pegamento (benomyl em 3/jan, na 'Natal') (Tabela 1), assim como o tratamento mais tardio com benomyl (aplicação do dia $7 /$ jan), foi eficiente nesta variedade. Na 'Pêra', este tratamento tardio resultou em pegamento superior a 3 vezes quando comparado com a testemunha, mesmo se considerar que o estágio de desenvolvimento dos botões, em $4 /$ jan, estivesse adiantado (predominância de cotonetes) e que não tenha ocorrido diferença estatística.

Não houve diferença estatística também entre uma e duas aplicações do fungicida. Na 'Natal', o tratamento com duas pulverizações reduziu significativamente a incidência de estilos necrosados e de cálices retidos, sem melhorar o pegamento (Tabela 1). Isto indica que, a partir de um certo pegamento, outros fatores passam a ser mais importantes na determinação da fixação das estruturas florais do que a PFC.

A distribuição dos estágios florais iniciais mostra que estágios de florada temporã com botões redondos fechados brancos, no início do desenvolvimento das pétalas ('Natal') ou quando do seu tamanho final ('Pêra') podem ser eficientemente tratados contra PFC na florada temporã. A faixa de estágios de florada entre estes extremos, portanto, pode ser considerada a ideal para tratamento. O período médio entre os estágios de botão redondo branco e cotonete foram de cerca de 7 dias para as condições de temperatura da florada temporã da 'Natal'. Observações na 'Pêra' indicam um comportamento semelhante, embora não tenha ocorrido número suficiente de botões florais redondos marcados para acompanhamento do seu desenvolvimento.

Pulverizações tratorizadas. As floradas pulverizadas foram às mesmas das aplicações manuais. As condições climáticas, portanto, foram muito favoráveis à doença. O estágio dos botões florais foi de predominantemente redondo fechado branco na 'Natal' e de fechado totalmente desenvolvido na 'Pêra'. Todos os tratamentos com fungicidas resultaram em aumento

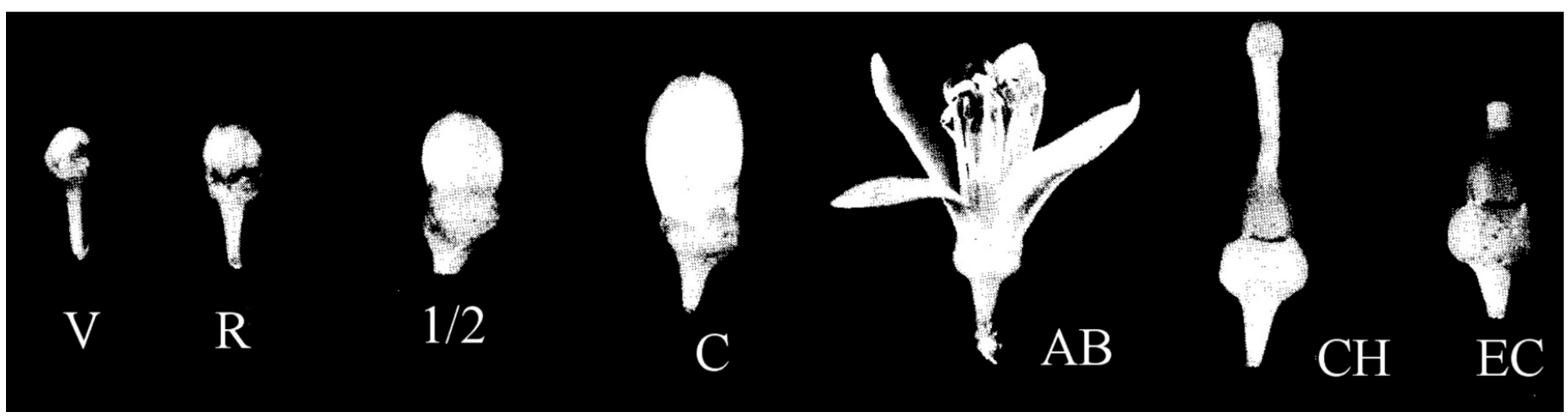

FIGURA 1 - Estádios florais de laranjeiras. (V): verde; (R): redondo; (1/2) do tamanho final; (C): cotonete; (AB): flor aberta; (CH): chumbinho; e (EC): estilo caído.

TABELA 1 - Efeito de aplicações de benomyl sobre botões florais de Laranja-'Natal' (Citrus sinensis [L.] Osbeck) contra a Podridão Floral dos Citros (Colletotrichum acutatum).

\begin{tabular}{lcccc}
\hline & \multicolumn{4}{c}{$\%$ dos botões florais } \\
\cline { 2 - 5 } Tratamentos $^{\mathbf{y}}$ & $\begin{array}{c}\text { Sintoma } \\
\text { nas pétalas }\end{array}$ & $\begin{array}{c}\text { Estilo } \\
\text { Necrosado }\end{array}$ & $\begin{array}{c}\text { Cálices } \\
\text { retidos } \\
\text { (estrelinhas) }\end{array}$ & Pegamento \\
\hline Testemunha & $85 \mathrm{a}$ & $59 \mathrm{a}$ & $80 \mathrm{a}$ & $1 \mathrm{~b}$ \\
Benomyl 3/jan & $62 \mathrm{~b}$ & $27 \mathrm{~b}$ & $53 \mathrm{~b}$ & $16 \mathrm{a}$ \\
Benomyl 3 e 11/jan & $66 \mathrm{ab}$ & $12 \mathrm{c}$ & $29 \mathrm{c}$ & $18 \mathrm{a}$ \\
Benomyl 7/jan & $75 \mathrm{ab}$ & $28 \mathrm{~b}$ & $54 \mathrm{~b}$ & $16 \mathrm{a}$ \\
\hline
\end{tabular}

Médias seguidas da mesma letra nas colunas não diferem significativamente entre si, pelo teste de Duncan $(P<0,01)$.

${ }^{y}$ Benomyl a 0,5 g.L $\mathrm{L}^{-1}$. Pulverizações manuais de ramos marcados.
TABELA 2 - Efeito de aplicações de benomyl sobre botões florais de Laranja-'Pêra' (Citrus sinensis [L.] Osbeck) contra a Podridão Floral dos Citros (Colletotrichum acutatum).

\begin{tabular}{lcccc}
\hline & \multicolumn{4}{c}{$\%$ dos botões florais } \\
\cline { 2 - 5 } Tratamentos & $\begin{array}{c}\text { Sintoma } \\
\text { nas pétalas }\end{array}$ & $\begin{array}{c}\text { Estilo } \\
\text { Necrosado }\end{array}$ & $\begin{array}{c}\text { Cálices } \\
\text { retidos } \\
\text { (estrelinhas) }\end{array}$ & Pegamento \\
\hline Testemunha & $97 \mathrm{a}$ & $77 \mathrm{a}$ & $79 \mathrm{a}$ & $4 \mathrm{~b}$ \\
Benomyl 4/jan & $89 \mathrm{ab}$ & $89 \mathrm{a}$ & $75 \mathrm{a}$ & $16 \mathrm{a}$ \\
Benomyl 4 e 11/jan & $82 \mathrm{~b}$ & $59 \mathrm{~b}$ & $68 \mathrm{a}$ & $24 \mathrm{a}$ \\
Benomyl 6/jan & $97 \mathrm{a}$ & $79 \mathrm{a}$ & $79 \mathrm{a}$ & $14 \mathrm{ab}$ \\
\hline
\end{tabular}

Médias seguidas da mesma letra nas colunas não diferem significativamente entre si, pelo teste de Duncan $(P<0,01)$.

${ }^{\mathbf{y}}$ Benomyl a 0,5 g.L $\mathrm{L}^{-1}$. Pulverizações manuais de ramos marcados. 
TABELA 3 - Efeito de aplicações de benomyl contra Podridão Floral dos Citros (Colletotrichum acutatum) no pegamento e retenção do cálice (estrelinha) de botões florais em laranja-'Natal' (Citrus sinensis [L.] Osbeck).

\begin{tabular}{lcc}
\hline $\begin{array}{l}\text { Tratamentos }^{\mathbf{y}} \\
\text { (doses por 2.000 L) }\end{array}$ & \multicolumn{2}{c}{ \% dos botões florais marcados } \\
\cline { 2 - 3 } & Pegamento & Cálice retido \\
\hline Testemunha & $7 \mathrm{~b}$ & $74 \mathrm{a}$ \\
Benomyl 1kg, 4/jan & $20 \mathrm{a}$ & $33 \mathrm{~b}$ \\
Benomyl 1 kg, 4 e 11/jan & $21 \mathrm{a}$ & $12 \mathrm{c}$ \\
Benomyl 1 kg, 8/jan & $15 \mathrm{ab}$ & $31 \mathrm{~b}$ \\
\hline
\end{tabular}

Médias seguidas da mesma letra nas colunas não diferem significativamente entre si, pelo teste de Duncan $(P<0,01)$.

y Pulverizações tratorizadas, $6 \mathrm{~L} /$ planta.

TABELA 4 - Efeito de aplicações de fungicidas contra Podridão Floral dos Citros (Colletotrichum acutatum) no pegamento e retenção do cálice (estrelinha) de botões florais e no número total de frutos por planta em laranja-'Pêra' (Citrus sinensis [L.] Osbeck).

\begin{tabular}{lccc}
\hline Tratamentos $^{\mathrm{y}}$ & \multicolumn{3}{c}{$\%$ dos botões florais marcados } \\
\cline { 2 - 4 } (doses por 2.000 L) & Pegamento & Cálice retido & $\mathbf{N}^{\circ}$ de frutos por planta \\
\hline Testemunha & $18 \mathrm{~d}$ & $78 \mathrm{a}$ & $296 \mathrm{~b}$ \\
Benomyl 1 kg, 4/jan & $56 \mathrm{abc}$ & $738 \mathrm{a}$ \\
Benomyl 1 kg, 4 e 10/jan & $26 \mathrm{abc}$ & $40 \mathrm{bcd}$ & $757 \mathrm{a}$ \\
Benomyl 1 kg + captan 3,5 kg, 4/jan & $28 \mathrm{abc}$ & $40 \mathrm{bcd}$ & $746 \mathrm{a}$ \\
Benomyl 0,75 kg + captan 2,5 kg, 4/jan & $23 \mathrm{abc}$ & $49 \mathrm{bcd}$ & $745 \mathrm{a}$ \\
Benomyl 1 kg + captan 2,5 kg, 4/jan & $32 \mathrm{ab}$ & $45 \mathrm{bcd}$ & $563 \mathrm{a}$ \\
Idem anterior + benomyl 1 kg, 10/jan & $33 \mathrm{ab}$ & $31 \mathrm{~cd}$ & $634 \mathrm{a}$ \\
Benomyl 1,5 kg, 4/jan & $40 \mathrm{a}$ & $25 \mathrm{~d}$ & $640 \mathrm{a}$ \\
Benomyl 1,5 kg, 4 e 10/jan & $12 \mathrm{~cd}$ & $64 \mathrm{ab}$ & $695 \mathrm{a}$ \\
\hline
\end{tabular}

Médias seguidas da mesma letra nas colunas não diferem significativamente entre si, pelo teste de Duncan $(P<0,01)$.

y Pulverizações tratorizadas, $8 \mathrm{~L} /$ planta.

significativo do pegamento, e redução da incidência de cálices retidos (estrelinhas) (Tabelas 3 e 4). Na 'Pêra', o número de frutos em cada tratamento através da derriça foi também avaliado, resultando em significativo aumento para todos os tratamentos com fungicidas (Tabela 4).

Não houve diferença entre uma ou duas aplicações, entre tratamentos com doses diferentes, ou com a adição de captan ao benomyl. Observou-se, no entanto, como no caso das pulverizações manuais, uma tendência de redução de cálices retidos (estrelinhas) e aumento do pegamento à medida que se acrescenta uma aplicação, se adiciona outro fungicida, ou se aumenta à dose do benomyl.

\section{CONCLUSÕES}

1 - Benomyl é eficiente no controle da Podridrão Floral dos Citros em florada temporã, em doses de 0,5 a 0,75 g.L. $\mathrm{L}^{-1}$, utilizando-se de aplicações com volumes de calda de 6 a $8 \mathrm{~L} /$ planta.

2 - A resposta ao benomyl pode ser obtida em floradas temporãs de laranjeiras-doces tratadas nos estágios de predominância de botões redondos brancos até cotonetes.

\section{REFERÊNCIAS BIBLIOGRÁFICAS}

AGOSTINI, J.P., TIMMER, L.W. Populations dynamics and survival of strains of Colletotrichum gloeosporioides on citrus in Florida. Phytopathology, St. Paul, v.84, n.4, p.420-425, 1994.

COUTINHO, A., ROBERTO, S.R., LIMA, J.E.O. Podridão Floral dos Citros - estudos sobre sua incidência e controle. In: CONGRESSO BRASILEIRO DE FRUTICULTURA, 13., 1994, Salvador, Anais... p.369.

DENHAM, T.G. Citrus production and premature fruit drop disease in Belize. Pans, London, v.25, n.1, p.30-36, 1979.

FAGAN, H.J. Postbloom fruit drop of citrus, a new disease of citrus associated with a form of Colletotrichum gloeosporioides. Annual Applied Biology, London, v.91, n.1, p.13-20, 1979.

FEICHTENBERGER, E., MÜLLER, G.W., GUIRADO, N. Doenças dos citros (Citrus spp). In: KIMATI, H., AMORIM, L., BERGAMIN, A.F., CAMARGO, L.E.A., REZENDE, J.A.M. Manual de Fitopatologia. 3.ed. São Paulo: Agronômica Ceres, 1997, v.2, p.280-284

GOES, A., CRESTE, J.E. Uso do sistema integrado de diagnose e recomendação (DRIS) na avaliação de folhas de plantas cítricas com sintomas de queda prematura de frutos. Summa Phytopathologica, Jaboticabal, v.26, n.2, p.237-240, 2000.

MORETTO, K.C.K. Controle biológico da Queda Prematura dos Frutos Cítricos causado por Colletotrichum acutatum. 2000, 128f. Tese (Doutorado em Produção Vegetal) - Faculdade de Ciências Agrárias e Veterinárias, Universidade Estadual Paulista, Jaboticabal, 2000.

SONODA, R.M. An update on postbloom fruit drop in the Indian River area and results of a fungicide test on the disease. Proceedings of the Florida State Horticultural Society, Winter Haven, v.106, p.1-15, 1993.

TIMMER, L.W., AGOSTINI, J.P., ZITKO, S.E., ZULFIQAR, M. Postbloom fruit drop, a increasingly prevalent disease of citrus in the Americas. Plant Disease, St Paul, v.78, n.4, p.329334, 1994.

TIMMER, L.W., ZITKO, S.E. Timing of fungicides applications for control of postboom fruit drop of citrus in Florida. Plant Disease, St Paul, v.76, n.8, p.820-823, 1992. 